
pimenta. Horticultura Brasileira 24: 195-198.

\title{
Efeitos da adubação química e da calagem na nutrição de melissa e hor- telã-pimenta
}

\author{
Arie F Blank ${ }^{\mathbf{1}}$; Andréa dos S Oliveira ${ }^{\mathbf{1}}$; Maria de Fátima Arrigoni-Blank ${ }^{\mathbf{1}}$; Valdemar Faquin ${ }^{2}$ \\ ${ }^{1}$ UFS, Av. Marechal Rondon s/n, B. Rosa Elze, 49100-000 São Cristóvão-SE; 2 UFLA, C. Postal 37, 37200-000 Lavras-MG; E-mail: \\ arie.blank@terra.com.br
}

\begin{abstract}
RESUMO
O trabalho objetivou avaliar os efeitos da adubação química e da calagem no crescimento e na nutrição da melissa (Melissa officinalis) e hortelã-pimenta (Mentha piperita), com a técnica da diagnose por subtração. Os tratamentos foram: Completo (adubado com N, P, K, S, B, Cu, Fe e Zn+ calagem); Completo calagem; Completo N; Completo $\mathrm{P}$; Completo K; Completo S; Completo B; Completo Zn; Completo Fe e Testemunha (solo natural). Após 120 dias da semeadura a calagem e a adubação mostraram ser essenciais para o crescimento de melissa e hortelã-pimenta. No solo utilizado, a falta da calagem e dos nutrientes $\mathrm{N}$ e P causaram as maiores quedas ou ausência, no caso da falta de calagem em melissa, na produção de folhas, órgão usado na medicina popular e que contém os princípios ativos.
\end{abstract}

Palavras-chave: Melissa officinalis, Mentha piperita, nutrição mineral, elemento faltante.

\begin{abstract}
Effect of chemical fertilization and liming on lemon balm and peppermint nutrition

This work aimed to evaluate the effects of chemical fertilization and liming on growth and nutrition of lemon balm (Melissa officinalis) and peppermint (Mentha piperita), using the missing element technique. Treatments were: complete (fertilized with N, P, K, S, B, $\mathrm{Cu}, \mathrm{Fe}$ and $\mathrm{Zn}+$ liming); complete - liming; complete -N; complete $\mathrm{P}$; complete -K; complete -S; complete -B; complete -Zn; complete $\mathrm{Fe}$ and control (natural soil). The following results were obtained within 120 days after sowing: liming and fertilization are essential for lemon balm and peppermint growth. In the soil used in this experiment the nutrients which showed highest results on growth and nutrition of lemon balm and peppermint were $\mathrm{N}$ and $\mathrm{P}$, besides liming.
\end{abstract}

Keywords: Melissa officinalis, Mentha piperita, mineral nutrition, missing element.

\section{(Recebido para publicação em 17 de novembro de 2005 ; aceito em 31 de maio de 2006)}

$\mathrm{E}^{\mathrm{s}}$ studos de plantas medicinais voltam-se, preferencialmente, para o conhecimento de espécies que produzem fitofármacos desejados, ignorandose os processos genéticos e ambientais que influenciam a produção desses compostos químicos.

A melissa (Melissa officinalis L. Lamiaceae) é originária da Europa e da Ásia. É uma Herbácea de 0,20 a 0,80 m de altura, que prefere solos férteis e com alto teor de matéria orgânica (Martins et al., 1998). O óleo essencial das folhas é amplamente utilizado pela indústria farmacêutica por possuir propriedades antioxidativa, antimicótica, antivirótica e sedativa (Teske \& Trenttini, 1997).

O gênero Mentha (Lamiaceae), comumente conhecido como hortelãs, destaca-se pelo uso culinário e de chás com efeito medicinal, sendo conhecido pelo seu sabor característico e aroma refrescante. A espécie Mentha piperita L., conhecida como hortelã-pimenta, é produtora de óleo essencial rico em mentol e flavonóides, cujas aplicações nas indústrias farmacêuticas conferem- lhe grande importância econômica (Martins et al., 1998).

A maioria dos solos brasileiros são ácidos, o que pode causar dificuldades para a produção vegetal. Além da alta saturação de alumínio, que pode apresentar efeitos tóxicos para as plantas, os solos ácidos normalmente contêm baixo teor de cálcio e de magnésio trocáveis, cátions de grande importância para o desenvolvimento radicular. Uma das alternativas para minimizar esse problema é a correção da acidez através da prática da calagem (Goedert et al., 1991).

Na literatura há poucas informações sobre a fertilização química e exigências nutricionais de plantas medicinais, principalmente no Brasil. De maneira geral, os adubos químicos em poucos casos são prejudiciais aos teores de princípios ativos das plantas, quando usados dentro dos limites técnicos. Os aumentos de biomassa podem compensar uma redução do teor de fitofármacos, mas dependem da análise econômica, que deve ser feita em cada situação (Correa Júnior. et al., 1991).
A adubação química em guaco (Mikanea glomerata), foi benéfica quando na aplicação de nitrogênio mineral (60 g de sulfato de amônio por planta), resultou no aumento da produção de fitomassa em torno de seis vezes em relação à testemunha (Pereira et al., 1996). Em outro trabalho de fertilização em plantas medicinais, sobre o efeito de doses de NPK durante a fase de formação de mudas de jaborandi (Pilocarpus microphyllus Starf.), e utilizando-se como substrato uma mistura de terra preta, serragem curtida e esterco de curral, na proporção de $3: 1: 1$, concluiu se que as melhores produções de matéria seca foram conseguidas com as aplicações de 180 e 120 mg de nitrogênio e fósforo por quilograma de substrato, respectivamente (Brasil, 1996).

Para Atropa belladona a fertilização com 100; 35 e 120 kg/ha de N; P e K, respectivamente, resultou no aumento de $750 \mathrm{~kg} / \mathrm{ha}$ para $1700 \mathrm{~kg} / \mathrm{ha}$ de folhas secas e que não houve diferença significativa entre os teores de atropina, o que indica que a adubação química é vanta- 
Tabela 1. Altura de planta, peso da matéria seca de raiz, caule, folha, parte aérea e produção relativa da parte aérea de melissa (M. officinalis) e hortelã-pimenta (M. piperita). São Cristóvão-SE, UFS, 2004

\begin{tabular}{|c|c|c|c|c|c|c|}
\hline \multirow{3}{*}{ Tratamento } & \multirow{3}{*}{$\begin{array}{l}\text { Altura de } \\
\text { planta }(\mathrm{cm})\end{array}$} & \multicolumn{4}{|c|}{ Peso da matéria seca (g planta $\left.{ }^{-1}\right)$} & \multirow{2}{*}{$\begin{array}{c}\text { Produção } \\
\text { relativa } \\
(\%)\end{array}$} \\
\hline & & Raiz & Caule & Folha & $P$ arte aérea & \\
\hline & & \multicolumn{5}{|c|}{ Melissa officinalis } \\
\hline Completo & $25,30 \mathrm{a}$ & $1,221 \mathrm{bc}$ & 0,965 bc & $1,375 \mathrm{~cd}$ & $2,339 \mathrm{~cd}$ & 100,00 \\
\hline $\mathrm{N}$ & $9,03 \mathrm{c}$ & $0,009 \mathrm{c}$ & $0,038 \mathrm{c}$ & $0,773 d$ & $0,116 \mathrm{~cd}$ & 4,96 \\
\hline$P$ & $11,65 \mathrm{bc}$ & $0,048 \mathrm{c}$ & $0,039 \mathrm{c}$ & $0,127 d$ & $0,166 \mathrm{~cd}$ & 2,19 \\
\hline K & 25,08 a & $1,682 \mathrm{bc}$ & $0,862 \mathrm{bc}$ & $2,555 \mathrm{bc}$ & $3,418 \mathrm{bc}$ & 146,13 \\
\hline$S$ & 26,33 a & $0,871 \mathrm{bc}$ & $0,641 \mathrm{c}$ & $1,926 \mathrm{bcd}$ & $2,567 \mathrm{bcd}$ & 109,75 \\
\hline B & $18,65 a b$ & $0,985 \mathrm{bc}$ & $0,645 \mathrm{c}$ & $1,502 \mathrm{~cd}$ & $2,147 \mathrm{~cd}$ & 91,79 \\
\hline $\mathrm{Zn}$ & 27,18 a & $4,017 a b$ & $1,682 a b$ & $4,059 \mathrm{~b}$ & $5,741 a b$ & 245,45 \\
\hline $\mathrm{Fe}$ & $27,50 \mathrm{a}$ & $5,526 \mathrm{a}$ & $2,524 \mathrm{a}$ & 6,455 a & 8,979 a & 383,88 \\
\hline \multirow[t]{2}{*}{ Testemunha } & $3,30 \mathrm{c}$ & $0,001 \mathrm{c}$ & $0,011 \mathrm{c}$ & $0,017 \mathrm{~d}$ & $0,028 \mathrm{~d}$ & 1,20 \\
\hline & \multicolumn{6}{|c|}{ Mentha piperita } \\
\hline Completo & $54,03 \mathrm{ab}$ & $6,053 \mathrm{a}$ & 4,949 a & 4,873 a & $9,822 \mathrm{a}$ & 100,00 \\
\hline Cal & $26,95 \mathrm{bc}$ & $0,074 \mathrm{~b}$ & $1,182 \mathrm{bc}$ & $1,762 a b$ & $2,944 a b$ & 29,79 \\
\hline $\mathrm{N}$ & $26,13 \mathrm{bc}$ & $0,042 \mathrm{~b}$ & $0,235 \mathrm{c}$ & $0,264 b$ & $0,499 \mathrm{~b}$ & 5,08 \\
\hline$P$ & $5,00 \mathrm{c}$ & $0,010 \mathrm{~b}$ & $0,005 \mathrm{c}$ & $0,018 \mathrm{~b}$ & $0,023 b$ & 0,23 \\
\hline K & 57,48 a & $2,033 a b$ & $3,922 a b$ & $5,100 \mathrm{a}$ & $9,022 \mathrm{a}$ & 108,86 \\
\hline S & $40,00 \mathrm{ab}$ & $1,046 \mathrm{~b}$ & $1,717 a b c$ & $1,910 a b$ & $3,627 a b$ & 36,93 \\
\hline B & $53,15 a b$ & $2,672 a b$ & $2,534 a b c$ & $4,331 a b$ & $6,865 a b$ & 69,89 \\
\hline $\mathrm{Zn}$ & $54,13 a b$ & $4,059 a b$ & $3,409 a b c$ & $3,820 a b$ & $7,230 a b$ & 73,61 \\
\hline $\mathrm{Fe}$ & 57,95 a & $4,190 a b$ & $4,276 a b$ & $3,611 a b$ & 7,887 a & 80,30 \\
\hline Testemunha & $12,30 \mathrm{c}$ & $0,152 b$ & $0,064 \mathrm{c}$ & $0,457 \mathrm{~b}$ & $0,521 b$ & 5,30 \\
\hline
\end{tabular}

Médias seguidas pela mesma letra nas colunas não diferem entre si (Tukey 5\%). ${ }^{1} \mathrm{PR}(\%)=[\mathrm{MS}($ Trat. $) / \mathrm{MS}$ (completo) $] \times 100$.

josa para esta espécie (Montanari Júnior et al., 1993).

Arrigoni-Blank et al. (1999) concluíram que após 120 dias do transplantio, no cultivo da erva baleeira (Cordia verbenacea L.-Boraginaceae) em solos ácidos e de baixa fertilidade, a calagem e a adubação foram essenciais para o crescimento da plantas. Alem da prática da calagem, a adubação com N, K e $\mathrm{B}$ apresentou as maiores respostas quanto ao crescimento e à nutrição da espécie (Arrigoni-Blank et al., 1999).

$\mathrm{Na}$ busca de um sistema de produção economicamente viável, deve se implantar um programa de nutrição na cultura. No caso das espécies melissa (Melissa officinalis L.) e hortelã-pimenta (Mentha piperita L.), são escassos os trabalhos sobre suas exigências nutricionais e sobre os teores dos nutrientes na parte aérea. Assim, objetivou se no presente trabalho, avaliar os efeitos da adubação química e da calagem no crescimento e na nutrição de melissa e hortelã-pimenta.
O trabalho foi conduzido em casa de vegetação na Estação Experimental "Campus Rural da UFS" do Departamento de Engenharia Agronômica (DEA) da Universidade Federal de Sergipe (UFS), em vasos com 5,5 $\mathrm{dm}^{3}$. Foi utilizada amostra de solo de baixa fertilidade, retirada da camada subsuperficial $(20-40 \mathrm{~cm})$ de um Argissolo Vermelho-Amarelo (Embrapa, 1999), coletado no município de São Cristóvão (SE), com as seguintes características químicas: $\mathrm{pH}$ em água 4,$6 ; \mathrm{P}-0,7 \mathrm{mg} / \mathrm{dm}^{3}, \mathrm{~K}-0,12$ $\mathrm{cmol} / \mathrm{dm}^{3}$ (Mehlich 1); Ca+Mg - 0,77 $\mathrm{cmol} / \mathrm{dm}^{3}, \mathrm{Al}-0,90 \mathrm{cmol}_{\mathrm{c}} / \mathrm{dm}^{3}(\mathrm{KCl} 1$ mol/l) (EMBRAPA, 1979), $\mathrm{S} \quad \mathrm{SO}_{4}^{2}$ $0,76 \mathrm{cmol}_{\mathrm{c}} / \mathrm{dm}^{3}$ (Tedesco et al., 1985), $\mathrm{Zn}-0,97, \mathrm{Cu} \quad 0,66, \mathrm{Fe} \quad 82,9, \mathrm{Mn} 2,76$ $\mathrm{mg} / \mathrm{dm}^{3}$ (DTPA) e B (água quente) 0,10 $\mathrm{mg} / \mathrm{dm}^{3}$ (Jackson, 1970).

$\mathrm{O}$ delineamento experimental foi o de blocos ao acaso, com 10 tratamentos

\section{MATERIAL E MÉTODOS}

baseados na técnica do elemento faltante e oito repetições. Os tratamentos foram: (adubado com N, P, K, S, B, Cu, Fe e $\mathrm{Zn}+$ calagem); calagem ( $\mathrm{Cal}) ; \mathrm{N}(\mathrm{N})$; P ( P); K ( K); S ( S); B ( B); Zn ( Zn); Completo $\mathrm{Fe}(\mathrm{Fe})$ e (solo natural). Para o cálculo da necessidade de calagem, empregou se o método da saturação por bases, para elevar V a 70\%, usando se $\mathrm{CaCO}_{3}$ e $\mathrm{MgCO}_{3}$ p.a., na proporção equivalente Ca:Mg de 4:1 (Raij et al., 1996; Comissão..., 1999). Após a calagem, o solo foi mantido em incubação por 30 dias, com umidade de $70 \%$ do volume total de poros (VTP). Quando pertinente ao tratamento, foi aplicada a seguinte adubação básica de plantio: $67 \mathrm{mg} / \mathrm{dm}^{3} \mathrm{de} \mathrm{N} ; 150 \mathrm{mg} / \mathrm{dm}^{3} \mathrm{de} \mathrm{P}$; $97,5 \mathrm{mg} / \mathrm{dm}^{3}$ de $\mathrm{K} ; 40 \mathrm{mg} / \mathrm{dm}^{3}$ de $\mathrm{S} ; 0,5$ $\mathrm{mg} / \mathrm{dm}^{3}$ de B; $1,5 \mathrm{mg} / \mathrm{dm}^{3}$ de $\mathrm{Cu} ; 5,0$ $\mathrm{mg} / \mathrm{dm}^{3}$ de $\mathrm{Zn}$ e $5,0 \mathrm{mg} / \mathrm{dm}^{3}$ de Fe de solo (Arrigoni-Blank et al., 1999). As fontes dos nutrientes foram reagentes p.a. $\left(\mathrm{NH}_{4} \mathrm{NO}_{3}, \mathrm{NH}_{4} \mathrm{H}_{2} \mathrm{PO}_{4}, \mathrm{~K}_{2} \mathrm{SO}_{4}\right.$, $\mathrm{KH}_{2} \mathrm{PO}_{4}, \quad \mathrm{Ca}\left(\mathrm{H}_{2} \mathrm{PO}_{4}\right)_{2} \cdot \mathrm{H}_{2} \mathrm{O}$, $\mathrm{CaSO}_{4} \cdot 2 \mathrm{H}_{2} \mathrm{O}, \mathrm{MgSO}_{4} \cdot 7 \mathrm{H}_{2} \mathrm{O}, \mathrm{H}_{3} \mathrm{BO}_{3}$, $\mathrm{CuCl}_{2}, \mathrm{ZnCl}_{2}, \mathrm{Fe}$ EDTA).

Duas adubações de cobertura foram realizadas. A primeira, aos 40 dias após plantio (DAP), aplicaram se $15 \mathrm{mg} / \mathrm{dm}^{3}$ de $\mathrm{N}$, exceto nos tratamentos $\mathrm{N}$ e solo natural. A segunda, aos 70 DAP, constou da aplicação de: $20 \mathrm{mg}$ de N, 32,8 mg de Mg, 114 mg de Ca, 32 mg de S por $\mathrm{dm}^{3}$ de solo, exceto na testemunha e nos tratamentos onde foram omitidos.

Utilizou-se sementes comerciais de melissa e hortelã-pimenta. Foi realizada semeadura direta para as duas espécies, deixando apenas uma muda por vaso, sendo a umidade do solo mantida na capacidade de campo. As plantas foram colhidas aos 120 dias após a semeadura, separando se em folhas, caules e raízes, secas em estufa a $70^{\circ} \mathrm{C}$. Os teores dos macro e micronutrientes na matéria seca das folhas foram analisados quimicamente: $\mathrm{N}$, pelo método de Kjeldahl; P, K, Ca, S, Fe e Zn, por digestão nitricoperclórica com determinação no extrato feita como segue: $\mathrm{P}$ colorimetria; $\mathrm{K}$ fotometria de chama; $\mathrm{S}$ turbidimetria; $\mathrm{Ca}, \mathrm{Fe}$ e $\mathrm{Zn}$ espectrofotometria de absorção atômica; e B, por incineração e determinação colorimétrica pelo método da curcumina (Malavolta et al., 1989). 
Foram realizadas análises de variância da matéria seca de cada parte das plantas e do total da parte aérea, das raízes, altura de planta, teores dos macro e micronutrientes, e as médias comparadas pelo teste de Tukey, de acordo com Gomes (1985).

\section{RESULTADOS E DISCUSSÃO}

\section{Altura e peso de matéria seca}

Analisando as variáveis altura de planta de Melissa officinalis, observouse que, estatisticamente, houve diferenças significativas entre os tratamentos empregados (Tabela 1). Os tratamentos Completo e com omissão de K, S, Zn e Fe diferiram dos tratamentos com omissão de $\mathrm{N}$ e P e a Testemunha, tendo estes apresentando redução na altura de planta. Em trabalho realizado por Rodrigues et al. (2003) com manjericão (Ocimum sp.), testando diferentes concentrações de $\mathrm{P}$ e Mg, foi observado que, na medida em que era reduzida a concentração do $\mathrm{P}$, as plantas apresentavam sintomas visuais de deficiência, como folhas velhas mais escuras e sem brilho, seguidos por bronzeamento e queda das folhas. No presente estudo, quando foi omitida a calagem em melissa não houve sobrevivência da planta, permitindo inferir que a ausência de calagem é fator limitante para esta cultura.

A omissão de K, S, B, Zn, Fe e o tratamento completo proporcionaram as maiores médias de altura de planta e produção relativa, sendo que o tratamento com omissão de Fe apresentou os maiores valores em peso de matéria seca de raiz, caule, folha e parte aérea, não diferindo estatisticamente com -Zn para essas variáveis a exceção de peso de matéria seca de folha (Tabela 1). Isso permite inferir que esses elementos, no cultivo da melissa, não são limitantes no solo testado.

Na espécie Mentha piperita, para a variável altura de planta, o tratamento Completo e os com omissão de K, S, B, $\mathrm{Zn}$ e Fe diferiram estatisticamente dos tratamentos com omissão de $\mathrm{N}, \mathrm{P}$ e a testemunha. A omissão de $\mathrm{P}$ promoveu uma redução na altura de planta e no peso de matéria seca, reduzindo, conseqüentemente, a produção relativa. Rodrigues et al. (2004), realizando ex-

Tabela 2. Teores de macro e micronutrientes nas folhas no tratamento completo (Compl.) e nos respectivos tratamentos de omissão $(\mathrm{M})$, e produção relativa (PR) de matéria seca de folhas do tratamento de omissão em comparação ao completo, de melissa (M. officinalis) e hortelã-pimenta (M. piperita). São Cristóvão-SE, UFS, 2004.

\begin{tabular}{|c|c|c|c|c|c|c|}
\hline \multirow{3}{*}{ Nutriente } & \multicolumn{3}{|c|}{ Melissa officinalis } & \multicolumn{3}{|c|}{ Mentha piperita } \\
\hline & Compl. & $M$ & \multirow{2}{*}{ PR (\%) } & Compl. & $M$ & \multirow{2}{*}{$\mathrm{PR}^{1 /}(\%)$} \\
\hline & \multicolumn{2}{|c|}{$\left(\mathrm{g} \mathrm{kg}^{-1}\right)$} & & \multicolumn{2}{|c|}{$\left(\mathrm{g} \mathrm{kg}^{-1}\right)$} & \\
\hline $\bar{N}$ & 37,20 & -2 & -21 & $46,20 \mathrm{a}$ & $34,60 \mathrm{~b}$ & 74,89 \\
\hline$P$ & 4,18 & -2 & -21 & 3,05 & -21 & -2 \\
\hline K & $25,52 \mathrm{a}$ & $24,08 a$ & 94,36 & $24,56 \mathrm{a}$ & $18,30 \mathrm{a}$ & 74,51 \\
\hline $\mathrm{Ca}$ (calagem) & 16,60 & -2 & -21 & $16,12 \mathrm{a}$ & $11,89 \mathrm{a}$ & 73,76 \\
\hline Mg (calagem) & 6,81 & -2 & -21 & $9,34 \mathrm{a}$ & $5,29 a$ & 56,64 \\
\hline \multirow[t]{2}{*}{$\mathrm{S}$} & $3,91 \mathrm{a}$ & $3,68 \mathrm{a}$ & 94,12 & $3,21 \mathrm{a}$ & $2,64 \mathrm{a}$ & 82,24 \\
\hline & \multicolumn{2}{|c|}{$\left(\mathrm{mg} \mathrm{kg}^{-1}\right)$} & $(\%)$ & \multicolumn{2}{|c|}{$\left(\mathrm{mg} \mathrm{kg}^{-1}\right)$} & $(\%)$ \\
\hline$B$ & $58,45 \mathrm{a}$ & $42,13 a$ & 72,08 & $54,01 \mathrm{a}$ & $44,01 \mathrm{a}$ & 81,48 \\
\hline $\mathrm{Zn}$ & 29,64 a & $17,62 b$ & 59,45 & $27,84 \mathrm{a}$ & $19,34 \mathrm{a}$ & 69,47 \\
\hline $\mathrm{Fe}$ & 340,16 a & $220,10 a$ & 64,70 & $938,58 \mathrm{a}$ & $362,84 \mathrm{~b}$ & 38,66 \\
\hline
\end{tabular}

Médias seguidas pela mesma letra nas linhas, para cada espécie, não diferem entre si (Tukey $5 \%$ ). ${ }^{1 / \mathrm{PR}}(\%)=[\mathrm{MS}(\mathrm{M}) / \mathrm{MS}(\mathrm{Compl}$ ) $] \times 100$.

${ }^{2 / N a ̃ o ~ h o u v e ~ p r o d u c ̧ a ̃ o ~ d e ~ f o l h a s ~ s u f i c i e n t e s ~ p a r a ~ r e a l i z a c ̧ a ̃ o ~ d a ~ a n a ́ l i s e . ~}$

perimento com Mentha piperita L., testando diferentes concentrações de P, mostraram que, na medida em que se aumentou a concentração de $\mathrm{P}$, houve um aumento significativo no peso da matéria seca. Comportamento semelhante foi verificado com a omissão de $\mathrm{N}$.

Quanto à altura de planta, os tratamentos Completo e com omissão de S; $\mathrm{B}$; Zn; Fe e K proporcionaram as maiores médias, não diferindo estatisticamente. Na variável matéria seca da raiz, os tratamentos que omitiram os nutrientes N; P; S e Testemunha, apresentaram as menores médias. Em relação à parte aérea, tratamentos que omitiram $\mathrm{N}$ e $\mathrm{P}$ e Testemunha apresentaram as menores médias.

No tratamento com omissão de $\mathrm{P}$ houve uma drástica redução na altura da planta. Isto se deve ao fato de que o fósforo é um elemento que está diretamente ligado ao crescimento da planta, pois participa diretamente da construção das moléculas de DNA e RNA, responsáveis pela formação e diferenciação celular (Malavolta et al.,1989).

Na omissão de nitrogênio, o comportamento é semelhante ao tratamento com omissão de P. Como o N apresenta funções vitais nas plantas, como componente estrutural de macromoléculas e constituinte de enzimas, sua omissão resultou na redução da altura e do crescimento da planta.

\section{Teores dos nutrientes na matéria seca}

$\mathrm{Na}$ Tabela 2 apresenta-se os teores dos nutrientes nas folhas da melissa e hortelã-pimenta, nos tratamentos nos quais foram omitidos ( $\mathrm{M}$ ), comparativamente aos valores obtidos no tratamento completo (Compl.).

Para os tratamentos com a omissão de N, P e Calagem para melissa e de P para hortelã-pimenta o material produzido foi insuficiente, ou inexistente no caso do tratamento com a omissão de calagem em melissa, para a análise dos nutrientes (Tabela 2).

Observa-se que, de maneira geral, houve uma tendência de os teores dos nutrientes serem maiores no tratamento completo (Compl.), em relação àqueles onde foram omitidos ( $\mathrm{M}$ ), porém, nem sempre significativamente.

A omissão de $\mathrm{Zn}$ foi aquela que mais influenciou a produção relativa (PR) das folhas de melissa, indicando que o teor se encontrava em níveis abaixo do adequado. Ressalta se que foram observados sintomas visuais típicos de deficiência desse nutriente no tratamento no qual foi omitido ( M). Os tratamentos $\mathrm{K}, \mathrm{S}$, $\mathrm{B}$ e Fe não apresentaram diferença significativa em relação ao tratamento Completo quanto ao teor de $\mathrm{K}, \mathrm{S}, \mathrm{B}$ e $\mathrm{Fe}$, respectivamente, nas folhas de melissa.

A omissão de $\mathrm{N}$ e $\mathrm{Fe}$ foram que mais influenciaram na produção relativa (PR) 
das folhas de hortelã-pimenta, indicando que os teores se encontravam em níveis abaixo do adequado. Ressalta se que foram observados sintomas visuais típicos de deficiência desses nutrientes no tratamento no qual foi omitido. Os tratamentos com omissào de $\mathrm{K}, \mathrm{Ca}, \mathrm{Mg}, \mathrm{S}$, $\mathrm{B}$ e Zn não diferiram significativamente do tratamento Completo quanto aos seus teores nas folhas de hortelã-pimenta.

Conclui se que, com as observações feitas aos 120 dias da semeadura, no cultivo de melissa e hortelã-pimenta em solos ácidos e de baixa fertilidade, a calagem e a adubação são essenciais para seu crescimento. No solo utilizado, a falta da calagem e dos nutrientes $\mathrm{N}$ e $\mathrm{P}$ causaram as maiores quedas na produção de folhas, órgão usado na medicina popular e que contém os princípios ativos. A omissão de calagem em melissa não permitiu o crescimento das plantas.

\section{AGRADECIMENTOS}

Os autores agradecem ao ETENE/ FUNDECI/BNB pelo financiamento da pesquisa e CNPq pelas bolsas de produtividade do primeiro e último autor e de iniciação científica do segundo autor.

\section{REFERÊNCIAS}

ARRIGONI-BLANK MF; FAQUIN V; PINTO JEBP; BLANK AF; LAMEIRA OA. 1999. Adubação química e calagem em erva-baleeira (Cordia verbenacea). Horticultura Brasileira 17: 211-215.

BRASIL EC. 1996. Níveis de nitrogênio, fósforo e potássio na produção de mudas de jaborandi. REUNIÃO BRASILEIRA DE FERTILIDADE DO SOLO E NUTRIÇÃO DE PLANTAS, 22. Anais ... Manaus: SBCS. p. 666-667.

COMISSÃO DE FERTILIDADE DO SOLO DO ESTADO DE MINAS GERAIS. 1999. Recomendações para o uso de corretivos e fertilizantes em Minas Gerais. $5^{a}$ Aproximação. Viçosa: CFSEMG. 359p.

CORREA JUNIOR C; MINGLC; SCHEFFER MC. 1991. Cultivo de plantas medicinais, condimentares e aromáticas. Curitiba: EMATER-PR. 151p.

EMBRAPA-Serviço Nacional de Levantamento e Conservação dos Solos. 1979. Manual de métodos de análise de solo. Rio de Janeiro: EMBRAPA.

GOEDERT WJ; SOUZA DMG; SCOLARI DOG. 1991. Critérios para recomendação de calagem e adubação. In: OLIVEIRA AJ; GARRIDO WE; ARAUJO JD; LOURENÇO S (Coord.). Métodos de pesquisa em fertilidade do solo. Brasília: EMBRAPA-SEA. p. 363-392.

GOMES FP. 1985. Curso de estatística experimental. São Paulo: Nobel. 466p.

JACKSON ML. 1970. Analisis quimico de suelos. Barcelona: Omega. 662p.

MALAVOTA E. 1980. Elementos de nutrição mineral de plantas. Piracicaba: ESALQ. 251p.

MALAVOLTA E; VITTI GC; OLIVEIRA SA. 1989. Avaliação do estado nutricional das plantas: princípios e aplicações. Piracicaba: POTAFOS. 201p.
MARTINS ER; CASTRO DM; CASTELLANI DC; DIAS JE. 1998. Plantas Medicinais. Viçosa: Editora UFV. 220p.

MONTANARI JÚNIOR I; FIGUEIRA GM; MAGALHÃES PM; RODRIGUES N. 1993. Influência da fertilização NPK na biomassa e no teor de alcalóide de Atropa belladona Linn. Revista Brasileira de Fisiologia Vegetal 5: 71.

PEREIRAAMS; MENEZES JÚNIOR A; CÂMARA FLA; FRANÇA SC. 1996. Efeito da adubação na produção de biomassa de Mikanea glomerata (guaco). In: SIMPÓSIO DE PLANTAS MEDICINAIS DO BRASIL, 14. Anais.. Florianópolis: UFSC.

RAIJ JB; CANTARELLA H; QUAGGIO JA; FURLANI AMC (eds). 1996. Recomendações de adubação e calagem para o Estado de São Paulo. Campinas: IAC. 258p. (Boletim técnico, 100).

RODRIGUES CR; FAQUIN V; BERTOLUCCI SKV; PINTO JEBP; PEREIRA SP; SILVA S; CORRÊA MG; ANDRADE AT. 2003. Crescimento do manjericão em solução nutritiva sob diferentes concentrações de fósforo e magnésio. Horticultura Brasileira 21: Suplemento $\mathrm{CD}$.

RODRIGUES CR; FAQUIM V; TREVISAN D; PINTO JEBP; BERTOLUCCI SKV; RODRIGUES TM. 2004. Nutrição mineral, crescimento e teor de óleo essencial da menta em solução nutritiva sob diferentes concentrações de fósforo e épocas de colheita. Horticultura Brasileira 22: 573-578.

TEDESCO MJ; VOLKWEISS ST; BOHNEM H. 1985. Análise de solo, plantas e outros materiais. Porto Alegre: UFRGS. (Boletim técni$\mathrm{co}, 5)$.

TESKE M; TRENTTINI AMM. 1997. Compêndio de Fitoterapia. Paraná: Herbarium. 317p. 